\title{
Graphene-Based Strain Gauge on a Flexible Substrate
}

\author{
Mohammed Gamil', Hassan Nageh ${ }^{1}$, Ingy Bkrey ${ }^{1}$, \\ Sahour Sayed ${ }^{1}$, Ahmed M. R. Fath El-Bab ${ }^{2, \dagger}$, Koichi Nakamura ${ }^{1,3}$, \\ Osamu Tabata ${ }^{4}$ and Ahmed Abd El-Moneim ${ }^{1, *,+}$ \\ ${ }^{1}$ Materials Science and Engineering Department, Egypt-Japan University of Science and \\ Technology, New Borg El-Arab, Alexandria 21934, Egypt \\ ${ }^{2}$ Mechatronics and Robotics Department, Egypt-Japan University of Science and Technology, \\ New Borg El-Arab, Alexandria 21934, Egypt \\ ${ }^{3}$ Center for the Promotion of Interdisciplinary Education and Research, Kyoto University, \\ Kyoto Daigaku-Katsura, Kyoto 615-8540, Japan \\ ${ }^{4}$ Department of Micro Engineering, Kyoto University, \\ Kyoto Daigaku-Katsura, Kyoto 615-8540, Japan
}

(Received March 3, 2014; accepted April 1, 2014)

Key words: graphene oxide, graphene, strain gauge, gauge factor, flexible sensors

A flexible laser-reduced graphene oxide (LRGO) strain gauge formed on a polyethylene terephthalate (PET) substrate is fabricated using a new technique suitable for large-scale and low-cost mass production. The LRGO film is initially synthesized by drop-casting a graphene oxide (GO) solution on a flexible PET substrate, followed by the simultaneous reduction and patterning of the dried film with a $1.8 \mathrm{~W}$ carbon dioxide laser. The LRGO film is then characterized by X-ray diffraction (XRD), Fourier transform infrared (FTIR) spectroscopy, Raman spectroscopy, and scanning electron microscopy (SEM). A multilayer LRGO film with an estimated average thickness of 2.5 $\mu \mathrm{m}$ and good adhesion to the PET substrate is obtained. The measured gauge factor of the LRGO strain gauge is 61.5 .

\section{Introduction}

For roughly the past decade, the need to use flexible materials has augmented the challenges that researchers face when developing large-scale transparent and conducting films intended for use in flexible/stretchable devices such as displays, strain gauges, actuators, and micro-electromechanical systems (MEMS). ${ }^{(1-4)}$ Graphene-based flexible devices are attracting increasing attention owing to their excellent stretchability and flexibility, and graphene's ability to tolerate severe strain of up to $30 \%$, ${ }^{(5)}$ making it a

${ }^{*}$ Corresponding author: e-mail: ahmed.abdelmoneim@ejust.edu.eg

On leave from Mechanical Engineering Department, Faculty of Engineering, Assiut University, Assiut 71516, Egypt

¥On leave from Physical Chemistry Department, National Research Center, Cairo 12622, Egypt 
leading candidate for flexible devices. However, research on applications of graphene for flexible devices has been limited by constraints in producing large-scale graphene films on flexible substrates, namely, high synthesis temperatures, difficulty maintaining consistent quality between samples, fabrication cost, and difficulties associated with particular synthesis techniques.

Although graphene has so far been produced using several preparation techniques, mechanical exfoliation is currently the method of choice for producing high-quality graphene flakes. ${ }^{(6,7)}$ However, mechanical exfoliation is a nonreproducible and timeconsuming process that is limited to small-scale production. Recent advances in largescale synthesis of graphene films by chemical vapor deposition (CVD) on copper layers followed by assisted transfer methods ${ }^{(2,8)}$ are expected to enable various applications that employ substrates with areas up to approximately $10 \mathrm{~cm}^{2} .^{(1,8,9)}$ However, the lack of efficient etching and transfer methods limits the scale of production when using CVD techniques. Suspended graphene solutions can be prepared by reducing a graphene oxide solution prepared by the modified Hummers method, ${ }^{(10,11)}$ using any chemical or physical reduction method, ${ }^{(12,13)}$ but achieving a homogeneous and continuous film from a graphene solution after dispersal over a substrate by drop-casting, or even by spin coating, is challenging owing to the material's hydrophobicity. ${ }^{(14,15)}$

Another challenging issue when developing graphene-based flexible devices is patterning. Most previously reported graphene devices have been patterned using e-beam lithography owing their high resolution and writing freedom. However, e-beam lithography is unsuitable for batch fabrication of graphene-based devices that incorporate large flexible substrates owing to the high cost, substrate size constraints, and low writing speed. More importantly, e-beam irradiation may contaminate and even degrade the electrical and thermal conduction properties of the graphene owing to radiation-induced defects. $^{(16)}$

Lee et al. ${ }^{(3)}$ reported that the gauge factor of graphene grown by CVD over a copper film deposited on a $\mathrm{Si} / \mathrm{SiO}_{2}$ substrate is 6.1 at $1 \%$ applied strain, which is considerably higher than those of many metallic strain gauges. In a related study, Zheng et al. reported that the gauge factor of mechanically exfoliated graphene on a $\mathrm{Si} / \mathrm{SiO}_{2}$ substrate ranges from 10 to 15, depending on the number of layers in the graphene sheet.(17) Although this gauge factor is higher than that reported by Lee et al., the production method has a low yield, is messy, nonreproducible, time-consuming, and has poor scalability. Although Fu et al. ${ }^{(18)}$ demonstrated that monolayer graphene prepared by CVD and then transferred to a polydimethylsiloxane (PDMS) flexible substrate has a high sensitivity of 151 (gauge factor), the process of transferring the graphene to large-scale PDMS substrates is not feasible and the microfabrication process in this case relies on advanced techniques that make the cost of devices prohibitive.

Therefore, new research aims to develop alternative methods for the cost-effective fabrication of large flexible substrates for graphene-based devices such as flexible strain sensors. As a promising technique to meet these requirements, Strong et al. proposed a method of performing simultaneous reduction and patterning of predried graphene oxide (GO) prepared by the modified Hummers method and applied over a polyethylene terephthalate (PET) substrate, using a DVD drive laser source to fabricate flexible sensors. ${ }^{(19)}$ 
This is a simple, easy, and low-cost method of patterning GO films compared with electron-beam lithography or UV lithography. However, the quality of the laser-reduced GO prepared by this method is low. The high intensity of the D band observed in their graphene specimen suggests that carbon $\mathrm{sp}^{3}$ centers are still present after reduction. In addition, this process requires special sample preparation; the samples must be accurately formed as discs whose outer and inner diameters conform to the DVD standard so that they can be mounted in the DVD drive. The authors mentioned that the low quality of the produced graphene was due to insufficient reduction, a consequence of insufficient laser power, and that the quality would be improved by using a high-power laser source.

In this paper, we report the first production of a high-quality laser-reduced graphene oxide (LRGO) film with few defects, using a higher powered laser to simultaneously reduce and pattern a dried GO film dispersed over a flexible PET substrate. A commercially available laser printing machine was adopted, enabling reliable, high-throughput production while using larger patterning areas and a thicker film. Furthermore, in our method, the PET substrates are simultaneously patterned and reduced without special preparation or adjustment. In addition, the laser of the printing machine has sufficient power to allow the PET substrates to be accurately diced after reduction and patterning, which further lowers the production cost.

To demonstrate the feasibility of the proposed method, flexible LRGO-based strain gauges were formed on a PET substrate. The experimental methods, including GO preparation, fabrication, film characterization (GO, LRGO), and gauge factor measurement, are explained in $\S 2$. In $\S 3$, the production results, a discussion of film characterization, and gauge factor measurement results are presented. Conclusions are presented in $\S 4$.

\section{Experiments}

\subsection{Graphene oxide preparation}

High-quality GO was synthesized from purified natural graphite powder (Merck KGaA, Darmstadt, Germany, particle size $<50 \mu \mathrm{m}, 99.5 \%$, bulk density $20-30 \mathrm{gm} / 100$ $\mathrm{ml})$ by the modified Hummers method. ${ }^{(10,11)}$ The GO was diluted to a concentration of $3.7 \mathrm{mg} / \mathrm{mL}$ in double-distilled water and then sonicated for $30 \mathrm{~min}$ (Falc Instruments, Treviglio, Italy: power, $100 \mathrm{~W}$; frequency, $40 \mathrm{KHz}$ ), followed by centrifugation at $5000 \mathrm{rpm}$ for 20 min (Model EBA 20, Type 2002; Hettich Lab Technology, Tuttlingen, Germany).

\subsection{Fabrication}

The fabrication of the LRGO/PET strain gauge is shown schematically in Fig. 1. The GO solution was spread over a $0.1-\mathrm{mm}$-thick flexible PET substrate $12 \mathrm{~cm}$ in diameter by drop-casting at room temperature [Fig. 1(a)]. The GO film was then dried for $24 \mathrm{~h}$ at room temperature in air. LRGO patterns $\left(1 \times 2 \mathrm{~cm}^{2}\right.$ strips $)$ were formed on the predried GO film via direct laser reduction using a carbon dioxide $\left(\mathrm{CO}_{2}\right)$ laser of $10.6 \mu \mathrm{m}$ wavelength. The pattern was drawn using a laser printing machine (VLS 2.30; Universal Laser Systems, Scottsdale, AZ, USA) with an optical system having a focal length 


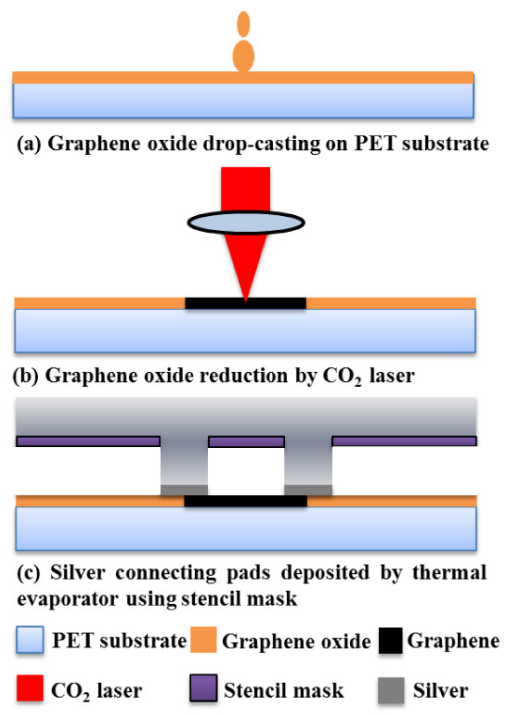

Fig. 1. (Color online) Fabrication processes for graphene-based flexible strain gauge.

of $51 \mathrm{~mm}$ at a pulse density of $393.7 \mathrm{p} / \mathrm{cm}(1000 \mathrm{PPI})$. On the basis of preliminary experiments, the optimal laser power was determined as $1.8 \mathrm{~W}$, the minimum required for reducing the prepared GO film (Fig. 2). Laser power above this value damaged the GO film and the PET substrate. At the end of the fabrication, silver electrodes were deposited onto the ends of the strips by thermal evaporation (Auto 306A Vacuum Coating System; Edwards Limited, Crawley, United Kingdom) using a stencil mask. Electrical resistance measurements were carried out after bonding wires to the silver electrodes.

\subsection{Film characterization}

The thickness of the GO film was measured using a stylus surface profiler (Dektak XT; Bruker Scientific Instruments, Billerica, MA, USA). To confirm the transformation of GO to LRGO, the GO and LRGO films were characterized by XRD (XRD-6100; Shimadzu Corporation, Kyoto, Japan), Raman spectroscopy (LabRAM HR-800; Horiba, Ltd., Kyoto, Japan), FTIR spectroscopy (Vertex 70; Bruker Scientific Instruments, Billerica, MA, USA), and SEM (JSM-6010LV; JEOL, Ltd., Tokyo, Japan).

\subsection{Gauge factor measurement}

The gauge factor of the LRGO-based strain gauge formed on the PET substrate was measured using a test rig (Fig. 3). The LRGO-based strain gauge and four commercial strain gauges (full Wheatstone bridge) were glued using a CN adhesive (Tokyo Sokki Kenkyujo Co., Ltd., Tokyo, Japan) near the fixed end of a 0.5 -mm-thick $(t) 15$-cmlong $(L)$ steel cantilever beam. When applying the glue, we ensured that all surfaces to be glued were clean, dry, and dust-free, then we dispensed a small amount of the glue 

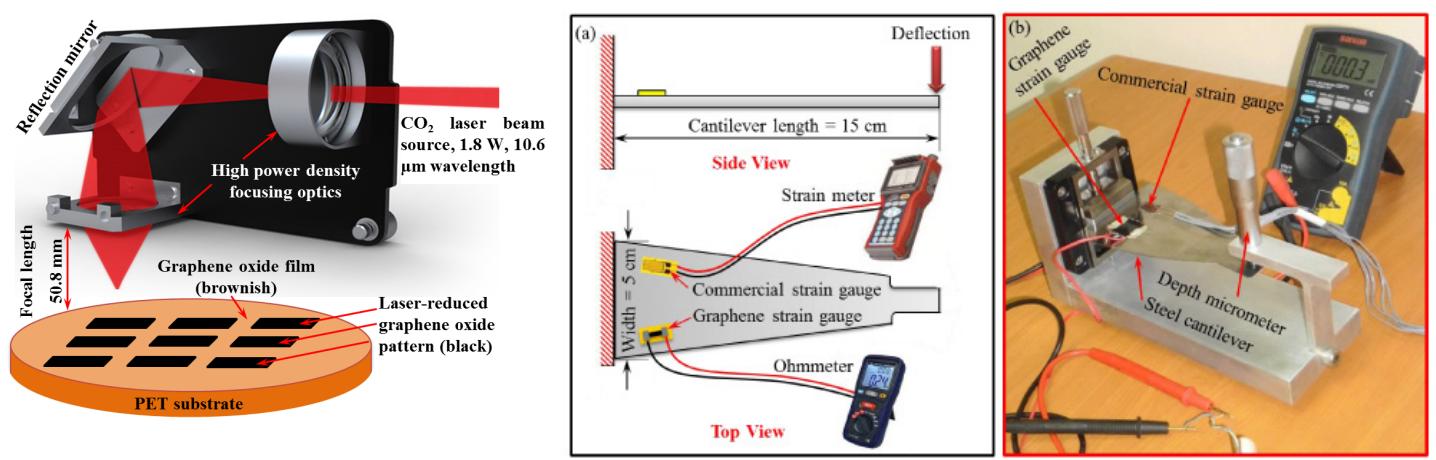

Fig. 2 (left). (Color online) Graphene oxide patterning and reduction by $\mathrm{CO}_{2}$ laser.

Fig. 3 (right). (Color online) Test rig for graphene-based strain gauge characterization: (a) schematic diagram and (b) image of test rig.

onto the steel cantilever surface. After that, the commercial and LRGO strain gauges were pressed firmly onto the glue for 2 min until the glue had nearly dried using nonsticking paper attached to the commercial strain gauges. A tapered beam shape was used so that homogenous uniaxial stress was generated on the beam surface. ${ }^{(20)}$ A downward force was applied to the free end of the cantilever beam using a depth micrometer. The LRGO/PET strain gauge and the commercial strain gauges were thus subjected to the same degree of uniaxial tensile strain owing to the downward deflection of the cantilever beam, causing changes in the electrical resistance of these devices. The strain $(\varepsilon)$ at any point $(x)$ in the triangular cantilever beam can be experimentally measured by the commercial strain gauges (full Wheatstone bridge) or can be theoretically calculated depending on the applied deflection $(\delta)$ at the free end of the beam using eq. (1).

$$
\varepsilon=(3 \delta x t) /\left(2 L^{3}\right)
$$

\section{Results and Discussion}

\subsection{Film characterization}

The measured surface profile of the GO film drop-cast on the PET substrate shown in Fig. 4 indicates that a rough surface with an average thickness of $2.5 \mu \mathrm{m}$ was formed.

XRD patterns of graphite powder, the deposited GO, and LRGO films are shown in Figs. 5(a)-5(c), respectively. In Fig. 5(a), the pattern of the graphite powder shows an intense peak at $26.4^{\circ}$ with an estimated interlayer distance of $3.38 \AA$. This interlayer distance is markedly expanded to $8.14 \AA$ following the oxidation of graphite to GO via the modified Hummers method [Fig. 5(b)]. After the application of infrared laser radiation to the GO film, a shallow peak appears at $25.92^{\circ}$ with an estimated interlayer distance of $3.43 \AA$ [Fig. 5(c)]. These changes indicate that the GO has been transformed into multilayer LRGO. ${ }^{(21-23)}$ 


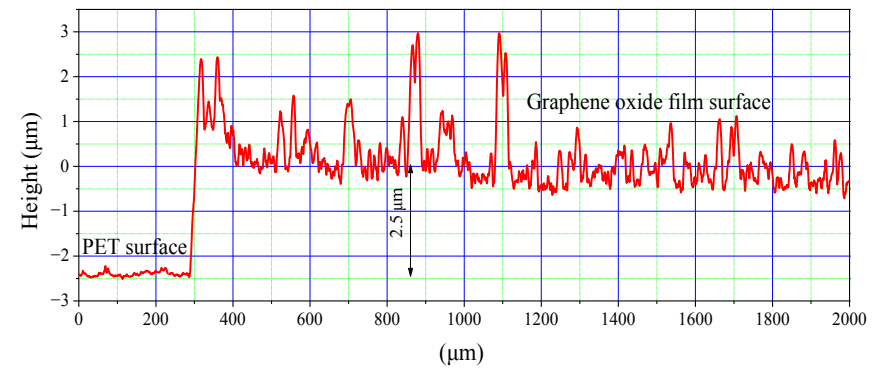

Fig. 4. (Color online) Profile of graphene oxide film cast on PET substrate obtained using a 2D stylus profiler.

(a)

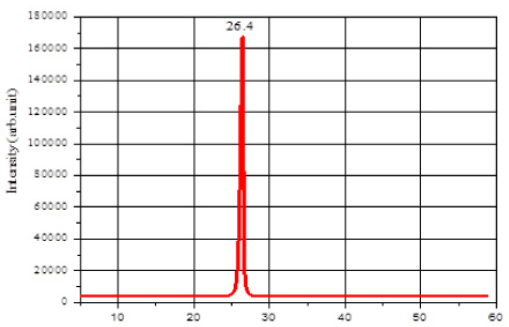

(b)

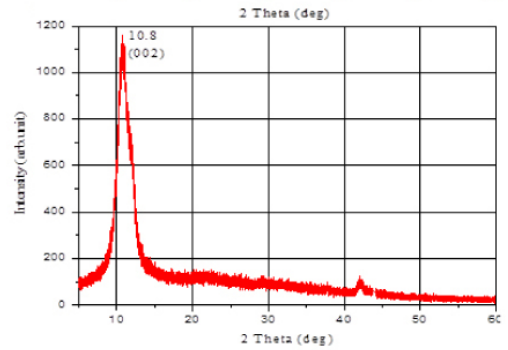

(c)

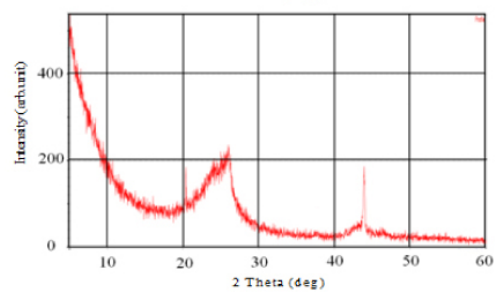

Fig. 5. (Color online) XRD patterns: (a) graphite powder, (b) graphene oxide, and (c) laserreduced graphene oxide film.

Figure 6 shows Fourier transform infrared (FTIR) spectra for GO [curve (1)] and LRGO [curve (2)]. The broad and intense peaks in the FTIR spectrum of the GO reveal evidence of stretching vibrations: $\mathrm{O}-\mathrm{H}$ absorption causes the peak at $3412 \mathrm{~cm}^{-1}$, and the peak at $1728 \mathrm{~cm}^{-1}$ is due to the $\mathrm{C}=\mathrm{O}$ stretching of carboxylic groups located at the edges of GO sheets. Stretching vibration peaks pertaining to various deformations were 


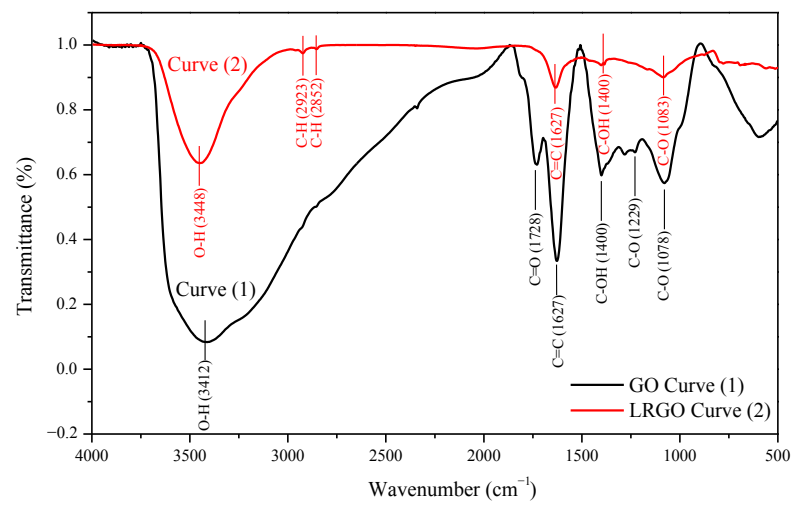

Fig. 6. (Color online) FTIR spectra for graphene oxide (black curve) and graphene (red curve).

observed: $\mathrm{OH}$ at $1400 \mathrm{~cm}^{-1}, \mathrm{C}-\mathrm{O}$ (epoxy) at $1229 \mathrm{~cm}^{-1}$, and $\mathrm{C}-\mathrm{O}$ (alkoxy) at $1078 \mathrm{~cm}^{-1}$. The formation of hydroxyl, carboxylic, epoxy, and alkoxy groups reflects the recorded interlayer expansion observed with XRD after the oxidation of graphite to GO. The peak at $1627 \mathrm{~cm}^{-1}$ corresponds to in-plane $\mathrm{C}=\mathrm{C}$ bonds and the skeletal vibration of the graphene sheets, further confirming the successful oxidation of the graphite. On the other hand, the FTIR spectrum for the LRGO indicates that the laser irradiation completely removes $\mathrm{C}=\mathrm{O}$ (carboxylic), $\mathrm{C}-\mathrm{O}$ (epoxy), and $\mathrm{C}-\mathrm{O}$ (alkoxy) groups, and partially eliminates hydroxyl groups as carbon-carbon bonding is simultaneously restored. It is worth mentioning that the remnants of hydroxyl groups are reported to be related to water molecules intercalated among LRGO layers; these remnants do not affect the hydrophobicity of the LRGO and usually improve its electrical conductivity. ${ }^{(12,24,25)}$

Figures 7(a) and 7(b) show Raman spectra for the GO and LRGO films deposited on the PET substrate, respectively. Figure 7(a) shows the two most prominent peaks necessary for GO characterization, the D band at $1344 \mathrm{~cm}^{-1}$ and the $\mathrm{G}$ band at $1604 \mathrm{~cm}^{-1}$. The absence of the $2 \mathrm{D}$ band around $2726 \mathrm{~cm}^{-1}$ after oxidation indicates that all graphite layers have been oxidized.

Figure 7(b) shows the three most prominent peaks for the LRGO, the G band at 1581 $\mathrm{cm}^{-1}$, the $2 \mathrm{D}$ band at $2702 \mathrm{~cm}^{-1}$, and the disorder-induced $\mathrm{D}$ band at $1360 \mathrm{~cm}^{-1}$. The $\mathrm{G}$ band results from in-plane vibrations of $\mathrm{sp}^{2}$ carbon atoms and is the most prominent feature of most graphitic materials. The $2 \mathrm{D}$ band is much more prominent in graphene than it is in bulk graphite. ${ }^{(26)}$ The D band is induced by defects in the graphene lattice and is not usually seen in highly ordered graphene layers. The intensity ratio of the G and $\mathrm{D}$ bands can be used to characterize the number of defects in a graphene sample.(27) The intensity of the Lorentzian G peak [Fig. 7(b)] is about three times larger than that of the D peak, confirming that the LRGO is disordered and includes defects. On the other hand, the profile of the $2 \mathrm{D}$ peak, as well as its intensity relative to the $\mathrm{G}$ peak, can be used to characterize the number of layers of a graphene film. ${ }^{(28)}$ The Lorentzian 2D peak is symmetric and its intensity is about half that of the G peak, indicating that LRGO with more than one layer was produced by our method. 


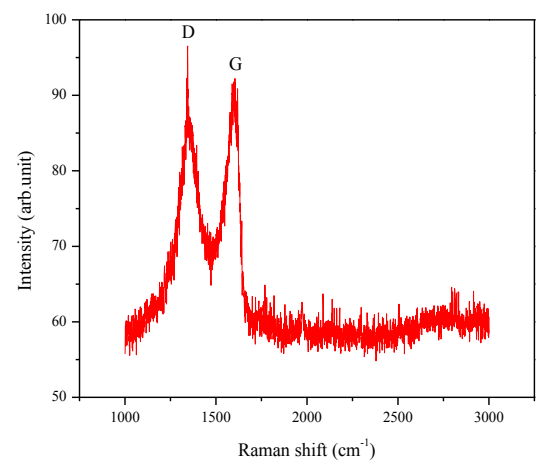

(a)

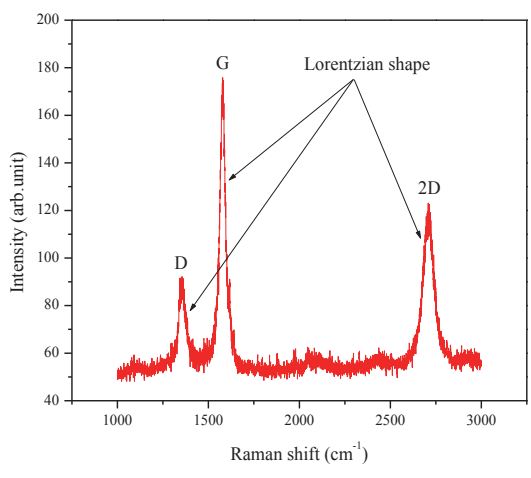

(b)

Fig. 7. (Color online) Raman spectra: (a) GO film and (b) LRGO film.

SEM images of the developed LRGO film at low and high magnifications are shown in Figs. 8(a) and 8(b), respectively. The image in Fig. 8(a) illustrates how laser irradiation has transformed the prepared coating of insulating GO (dark area in the lower part) into patterned conductive LRGO (lighter upper part with more contrast). The LRGO film shows no obvious cracks and is composed of overlapping flakes [Fig. 8(b)]. The absence of cracks enables the LRGO-based sensor to sensitively and reproducibly respond to strain.

\subsection{Gauge factor measurement results}

Figure 9 shows the effect of strain on the electrical resistance of the fabricated LRGO/PET strain gauge sensor. Under a strain-free condition, the average electrical resistance of the device was $1.174 \mathrm{k} \Omega$. The electrical resistance of the LRGO strain gauge sensor was then measured for different applied strains ranging from zero to $0.05 \%$, and each sequence of measurements was repeated 12 times. The measured resistance data for applied strains ranging from 0.01 to $0.04 \%$ showed good linearity for each sequence of measurement in the 12 measurements (Fig. 9). The average nonlinearity was $1.07 \%$, where the nonlinearity was calculated by dividing the output error from the linear fit at $0.025 \%$ strain by the output range. The reason for the nonlinearity observed in lower (0 to $0.01 \%)$ and higher $(0.04$ to $0.05 \%)$ strain ranges remains unclear. On the basis of the relationship between the changes in strain and electrical resistance in the range that had good linearity, the piezoresistive strain gauge factor for the LRGO-based sensor was calculated using eq. (2).

$$
G . F=(\Delta R / R) / \varepsilon
$$

Here $R$ is the strain-free LRGO resistance and $\Delta R$ is the change in the electrical resistance of the LRGO owing to the applied strain $\varepsilon$. From our calculations, the LRGO/PET strain gauge was found to have a gauge factor of 61.5 , which is far higher than those of commercial metal-alloy-based strain gauges and consistent with the simulation results 


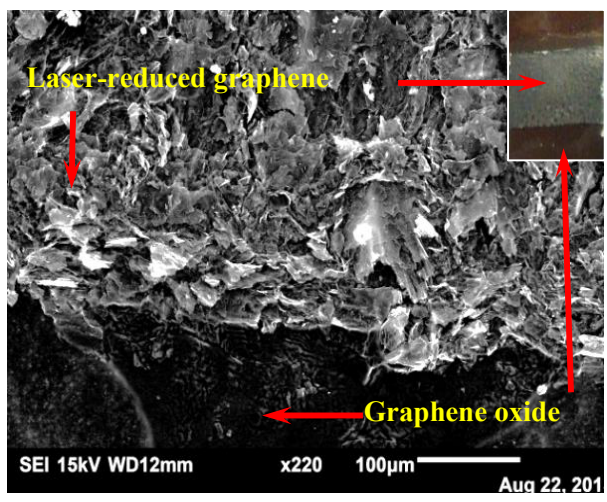

(a)

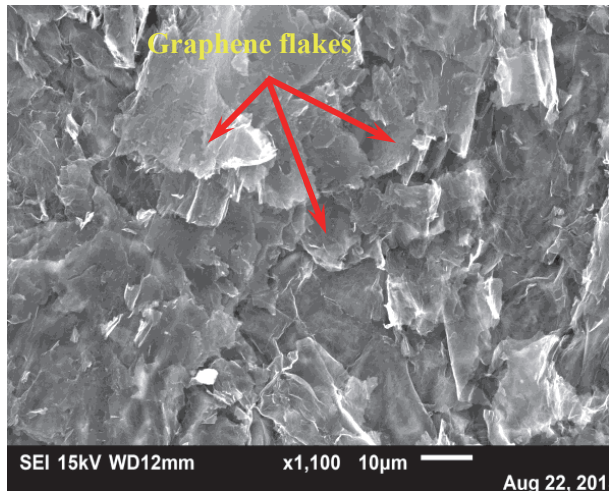

(b)

Fig. 8. (Color online) SEM images of laser-reduced graphene: (a) low magnification and (b) high magnification.

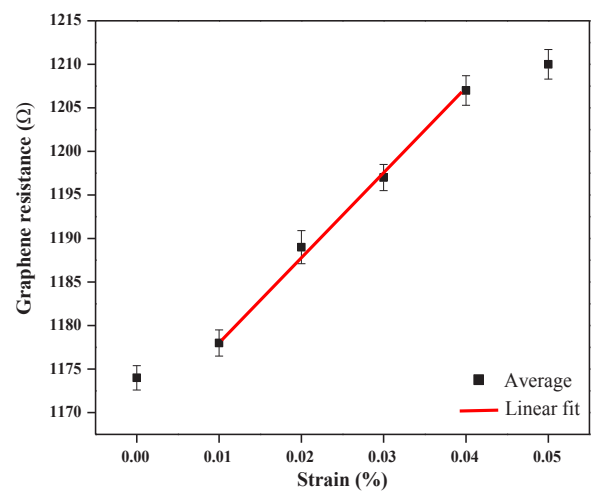

Fig. 9. (Color online) Changes in graphene electrical resistance (average with upper and lower limits of one standard deviation) under different strains.

obtained by Gamil et al., ${ }^{(29)}$ but less than those of strain gauges based on single-layer graphene films fabricated using complex and expensive high-vacuum CVD methods. ${ }^{(3)}$ This highlights the practicality of the proposed method, which can be used to easily fabricate strain gauge sensors that are much more sensitive than commercial metallic strain gauges and more feasible than silicon single-crystal strain gauges fabricated using expensive microfabrication techniques.

Figure 10 shows photographs of the LRGO/PET strain gauge and its fixation to the test rig. The LRGO/PET strain gauge showed no visual cracks or exfoliation from the PET substrate in the LRGO layer or in the GO film after being subjected to 20 repeated manual bending cycles, which reflects the good adhesion between the LRGO and GO on the PET substrate. 


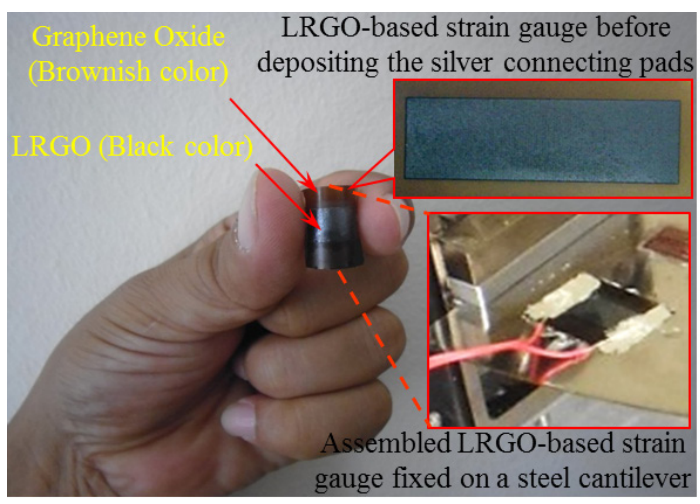

Fig. 10. (Color online) Photograph of the graphene-based strain gauge on the PET substrate.

\section{Conclusions}

We proposed and verified a fabrication method for producing patterned multilayer LRGO films on large flexible PET substrates using a cost-effective approach. The proposed method, which uses a relatively high-power carbon dioxide laser to produce an LRGO film, is simple, economical, and suited for mass production. A multilayer LRGO film with an estimated average thickness of $2.5 \mu \mathrm{m}$ and good adhesion to the PET substrate was obtained. The feasibility of the proposed method was demonstrated by successfully fabricating a flexible LRGO/PET strain gauge sensor. The measured gauge factor of this strain gauge was 61.5, much higher than those of commercial sensors based on metallic alloys. On the basis of these results, we conclude that the proposed method offers a new and versatile means of fabricating large-scale flexible LRGO/PET devices at a low cost.

\section{Acknowledgements}

This research was conducted as a Bilateral Joint Research Project funded by the Science and Technology Development Fund (STDF) in Egypt and Japan Society for the Promotion of Science (JSPS). The first author is sincerely grateful to the Mission SectorMOHE for providing a Ph.D. degree scholarship fund and to the Japan International Cooperation Agency (JICA) for their support of this work.

\section{References}

1 K. S. Kim, Y. Zhao, H. Jang, S. Y. Lee, J. M. Kim, K. S. Kim, J.-H. Ahn, P. Kim, J.-Y. Choi and B. H. Hong: Nature 457 (2009) 706.

2 X. Li, W. Cai, J. An, S. Kim, J. Nah, D. Yang, R. Piner, A. Velamakanni, I. Jung and E. Tutuc: Science 324 (2009) 1312. 
3 Y. Lee, S. Bae, H. Jang, S. Jang, S.-E. Zhu, S. H. Sim, Y. I. Song, B. H. Hong and J.-H. Ahn: Nano Lett. 10 (2010) 490.

4 S.-H. Bae, Y. Lee, B. K. Sharma, H.-J. Lee, J.-H. Kim and J.-H. Ahn: Carbon 51 (2012) 236.

5 Y. Wang, R. Yang, Z. Shi, L. Zhang, D. Shi, E. Wang and G. Zhang: ACS Nano 5 (2011) 3645 .

6 K. S. Novoselov, D. Jiang, F. Schedin, T. Booth, V. Khotkevich, S. Morozov and A. Geim: Proc. Natl. Acad. Sci. U.S.A. 102 (2005) 10451.

7 K. S. Novoselov, A. K. Geim, S. Morozov, D. Jiang, Y. Zhang, S. Dubonos, I. Grigorieva and A. Firsov: Science 306 (2004) 666.

8 Q. Yu, J. Lian, S. Siriponglert, H. Li, Y. P. Chen and S.-S. Pei: Appl. Phys. Lett. 93 (2008) 113103.

9 A. Reina, X. Jia, J. Ho, D. Nezich, H. Son, V. Bulovic, M. S. Dresselhaus and J. Kong: Nano Lett. 9 (2008) 30.

10 W. S. Hummers Jr. and R. E. Offeman: J. Am. Chem. Soc. 80 (1958) 1339.

11 J. Zhao, S. Pei, W. Ren, L. Gao and H.-M. Cheng: ACS Nano 4 (2010) 5245.

12 S. Stankovich, D. A. Dikin, R. D. Piner, K. A. Kohlhaas, A. Kleinhammes, Y. Jia, Y. Wu, S. T. Nguyen and R. S. Ruoff: Carbon 45 (2007) 1558.

13 H.-W. Chang, Y.-C. Tsai, C.-W. Cheng, C.-Y. Lin and P.-H. Wu: Electrochem. Commun. 23 (2012) 37.

14 O. Leenaerts, B. Partoens and F. Peeters: Phys. Rev. B 79 (2009) 235440.

15 S. Wang, Y. Zhang, N. Abidi and L. Cabrales: Langmuir 25 (2009) 11078.

16 D. Teweldebrhan and A. A. Balandin: Appl. Phys. Lett. 94 (2009) 013101.

17 X. Zheng, X. Chen, J.-K. Kim, D.-W. Lee and X. Li: Journal of Micro/Nanolithogr. MEMS MOEMS 12 (2013) 013009.

18 X.-W. Fu, Z.-M. Liao, J.-X. Zhou, Y.-B. Zhou, H.-C. Wu, R. Zhang, G. Jing, J. Xu, X. Wu and W. Guo: Appl. Phys. Lett. 99 (2011) 213107.

19 V. Strong, S. Dubin, M. F. El-Kady, A. Lech, Y. Wang, B. H. Weiller and R. B. Kaner: ACS Nano 6 (2012) 1395.

20 X. Chen, X. Zheng, J.-K. Kim, X. Li and D.-W. Lee: J. Vac. Sci. Technol. B 29 (2011) 06FE01.

21 H.-K. Jeong, Y. P. Lee, R. J. Lahaye, M.-H. Park, K. H. An, I. J. Kim, C.-W. Yang, C. Y. Park, R. S. Ruoff and Y. H. Lee: J. Am. Chem. Soc. 130 (2008) 1362.

22 T. Szabó, O. Berkesi, P. Forgó, K. Josepovits, Y. Sanakis, D. Petridis and I. Dékány: Chem. Mater. 18 (2006) 2740.

23 H. J. Shin, K. K. Kim, A. Benayad, S. M. Yoon, H. K. Park, I. S. Jung, M. H. Jin, H. K. Jeong, J. M. Kim and J. Y. Choi: Adv. Funct. Mater. 19 (2009) 1987.

24 M. Wojtoniszak, X. Chen, R. J. Kalenczuk, A. Wajda, J. Lapczuk, M. Kurzewski, M. Drozdzik, P. K. Chu and E. Borowiak-Palen: Colloids Surf., B 89 (2012) 79.

25 D. Li, M. B. Mueller, S. Gilje, R. B. Kaner and G. G. Wallace: Nat. Nanotechnol. 3 (2008) 101.

26 M. Pimenta, G. Dresselhaus, M. S. Dresselhaus, L. Cancado, A. Jorio and R. Saito: Phys. Chem. Chem. Phys. 9 (2007) 1276.

27 Y. Wang, Z. Ni, Z. Shen, H. Wang and Y. Wu: Appl. Phys. Lett. 92 (2008) 043121.

28 Z. Ni, Y. Wang, T. Yu and Z. Shen: Nano Res. 1 (2008) 273.

29 M. Gamil, K. Nakamura, A. F. El-Bab, O. Tabata and A. A. EL-Moneim, Modell. Numer. Simul. Mater. Sci. 3 (2013) 117. 